\title{
Community- and Hospital-Acquired Acute Kidney Injury in COVID-19: Different Phenotypes and Dismal Prognosis
}

\author{
Armando J. Martínez-Rueda Rigoberto D. Álvarez R. Angélica Méndez-Pérez \\ Dheni A. Fernández-Camargo Jorge E. Gaytan-Arocha Nathan Berman-Parks \\ Areli Flores-Camargo Roque A. Comunidad-Bonilla Juan M. Mejia-Vilet \\ Mauricio Arvizu-Hernandez Juan C. Ramirez-Sandoval Ricardo Correa-Rotter \\ Olynka Vega-Vega \\ Department of Nephrology and Mineral Metabolism, Instituto Nacional de Ciencias Médicas y Nutrición Salvador \\ Zubirán, Mexico City, Mexico
}

\section{Keywords}

SARS-CoV-2 · COVID-19 · Acute kidney injury · Mortality

\begin{abstract}
Introduction: Acute kidney injury (AKI) is common in coronavirus disease 2019 (COVID-19). It is unknown if hospitalacquired AKI (HA-AKI) and community-acquired AKI (CA-AKI) convey a distinct prognosis. Methods: The study aim was to evaluate the incidence and risk factors associated with both CA-AKI and HA-AKI. Consecutive patients hospitalized at a reference center for COVID-19 were included in this prospective cohort study. Results: We registered 349 (30\%) AKI episodes in 1,170 hospitalized patients, 224 (19\%) corresponded to $\mathrm{CA}-\mathrm{AKI}$, and 125 (11\%) to HA-AKI. Compared to patients with $\mathrm{HA}-\mathrm{AKI}$, subjects with CA-AKI were older (61 years [IQR 49-70] vs. 50 years [IQR 43-61]), had more comorbidities (hypertension [44 vs. $26 \%$ ], CKD [10 vs. $3 \%$ ]), higher Charlson Comorbidity Index (2 points [IQR 1-4] vs. 1 point [IQR 0-2]), and presented to the emergency department with more severe disease. Mortality rates were not different between CAAKI and HA-AKI (119 [53\%] vs. 63 [50\%], $p=0.66)$. In multi-
\end{abstract}

variate analysis, CA-AKI was strongly associated to a history of CKD (OR 4.17, 95\% Cl 1.53-11.3), hypertension (OR 1.55, 95\% Cl 1.01-2.36), Charlson Comorbidity Index (OR 1.16, 95\% Cl 1.02-1.32), and SOFA score (OR 2.19, 95\% Cl 1.872.57). HA-AKI was associated with the requirement for mechanical ventilation (OR 68.2, 95\% Cl 37.1-126), elevated troponin I (OR 1.95, 95\% Cl 1.01-3.83), and glucose levels at admission (OR 1.05, 95\% Cl 1.02-1.08).Discussion/Conclusions: CA-AKI and HA-AKI portend an adverse prognosis in COVID-19. Nevertheless, CA-AKI was associated with a higher comorbidity burden (including CKD and hypertension), while HA-AKI occurred in younger patients by the time severe multiorgan disease developed. @ 2021 S. Karger AG, Basel

\section{Introduction}

The coronavirus disease 2019 (COVID-19), caused by the severe acute respiratory syndrome coronavirus 2 (SARS-CoV-2) has spread to all regions of the world since December 2019 [1]. Due to the expression of the angio-

$\begin{aligned} & \text { karger@karger.com } \\ & \text { www.karger.com/bpu }\end{aligned}$
Karger ${ }^{\prime /}$

Olynka Vega-Vega

Department of Nephrology and Mineral Metabolism, Instituto Nacional de Ciencias Médicas y Nutrición Salvador Zubirán

Vasco de Quiroga 15, Colonia Seccion XVI, Mexico City 14080 (Mexico) olynkavega@ hotmail.com 
tensin-converting enzyme 2 in tubular cells and podocytes [2], a high incidence of kidney failure was expected to occur due to direct kidney viral damage.

Although initial postmortem biopsy series demonstrated the presence of virus-like particles in the kidney's tubular structures $[3,4]$, larger and more recent reports using electron microscopy and/or immunohistochemistry have not detected the virus in the kidney tissue [5-8]. Besides direct cytopathic damage from the virus, several other mechanisms have been proposed to underlie kidney injury in COVID-19, including effects on the renin-angiotensinaldosterone system, hypovolemia, and hypotension with ensuing ischemia, coagulopathy and microthrombosis, the cytokine storm, and septic kidney injury [9-11].

Acute kidney injury (AKI) seems to be common in COVID-19 [12-14], especially in patients admitted to the critical care unit (CCU) [14-16]. As it occurs in other critical illnesses, AKI in COVID-19 is associated with worsening of outcomes, including respiratory failure, inhospital mortality, and length of hospital stay [14, 15]. The KDIGO guidelines for AKI evaluation emphasize the distinction between "hospital-acquired" AKI (HA-AKI), and the AKI already present at patient admission or "community-acquired AKI" (CA-AKI) [17]. In a recent meta-analysis, CA-AKI was significantly associated with a higher AKI severity stage, yet lower mortality, when compared to HA-AKI [18].

It remains unexplored if CA-AKI or HA-AKI represents different entities with distinct prognosis in $\mathrm{CO}$ VID-19 patients. This study aimed to determine the frequency, risk factors, and outcomes for CA-AKI and HA$\mathrm{AKI}$ in a cohort of COVID-19 patients.

\section{Materials and Methods}

\section{Study Population}

This is a single-center, prospective, observational cohort study performed at the Instituto Nacional de Ciencias Médicas y Nutrición Salvador Zubirán, a reference center designated to treat exclusively patients with COVID-19 in Mexico City since March 16, 2020. The study was approved by the local Human Research and Ethics Boards (reference number NMM-3325-20-20-1). Written informed consent was waived due to the observational nature of the study.

All consecutive patients $>18$ years old admitted between March 16 and July 16 were evaluated. A prospective database collected demographic, medical history, home medications, comorbidities, clinical, laboratory, and CT-scan findings at patient admission, the requirement for attendance into a CCU, need for intubation, and/ or vasopressors, urine tests, and patient outcomes. Urine testing included urine electrolytes and urinalysis by automated microscopy, performed within $24 \mathrm{~h}$ of the AKI detection.
All the included patients had severe COVID-19 pneumonia diagnosed by a typical chest computed tomography and/or a positive test by the PCR of a nasopharyngeal sample for SARS-CoV-2 with requirement for supplementary oxygen. We excluded patients who remained hospitalized by July 16, patients admitted and discharged voluntarily or re-admitted after discharge, patients transferred to another institution for which we were unable to obtain data, and cases with prior kidney transplantation or end-stage kidney disease.

\section{Variable Definitions}

AKI was defined and staged according to the Kidney Disease Improving Global Outcomes (KDIGO) criteria [17]. Baseline serum creatinine $(\mathrm{sCr})$ was defined as the mean $\mathrm{sCr}$ in the previous 6 months before hospitalization, or the minimum sCr value during hospitalization if previous values were unavailable [19]. As urine output was absent in many cases, this criterion was not considered for AKI assessment. CA-AKI was defined as the presence of AKI at admission or developing AKI $<48 \mathrm{~h}$ after hospital admission. HA-AKI was defined as any AKI documented beyond $48 \mathrm{~h}$ of hospital admission.

CKD was defined by the KDIGO CKD guidelines based on previously documented findings or during the evaluation for $\mathrm{CO}$ VID-19 [20]. The estimated glomerular filtration rate was calculated using the Chronic Kidney Disease Collaboration creatinine equation [21]. Recovery from AKI was operationally defined as a reduction in peak AKI stage, and further refined by a reduction in sCr level to a sCr level $<1.5$ times baseline [22]. The Charlson Comorbidity Index [23] was obtained to summarize comorbidity information.

\section{Outcomes}

The primary outcome was to describe the frequency and risk factors associated with CA-AKI and HA-AKI. Secondary outcomes included the requirement for admission to a CCU, final hospital disposition (discharge or death), and renal function recovery.

\section{Statistical Analysis}

Continuous variable distribution was assessed by the Kolmogorov-Smirnov test. Descriptive statistics are expressed as median (interquartile range [IQR]) for continuous variables and number (\%) for categorical variables. Between-group comparisons were performed by nonparametric ANOVA with Dunnett's correction, or $\chi^{2}$, as appropriate.

Logistic regression was used to define the factors associated with CA-AKI and HA-AKI. Age, sex, and all variables with a $p$ value $<0.05$ on bivariate analysis were included for the forward stepwise multivariate modeling. There were $<1 \%$ missing values for any variable. In the case of missing data, variables were imputed by using multiple imputations. The final models were evaluated by the Hosmer-Lemeshow test. All statistical tests were two-sided, and a $p$ value $<0.05$ was considered as statistically significant. All analyses were performed using SPSS 24.0 (IBM, Armonk, NY, USA) and GraphPad Prism 6.0 (GraphPad Software, San Diego, CA, USA). 
Fig. 1. Flow chart of the patients included in this study. HA-AKI, hospital-acquired acute kidney injury; CA-AKI, communityacquired acute kidney injury; COVID-19, coronavirus disease 2019.

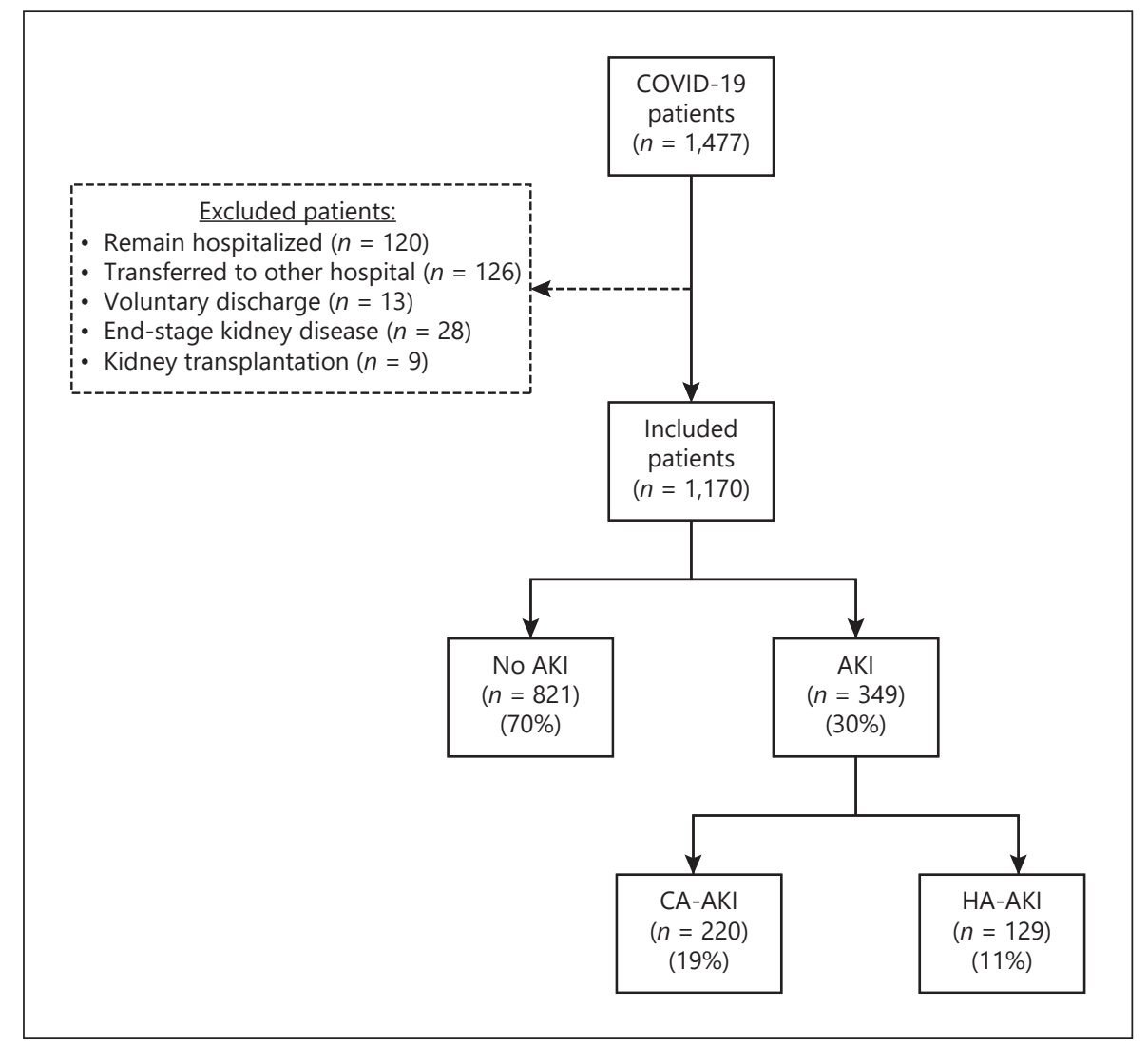

\section{Results}

From March 16 to July 16, 2020, 1,534 patients were admitted to our center of which 1,477 had COVID-19 diagnosis. Of these, we included 1,170 patients for this analysis. All patients had severe COVID-19 associated pneumonia requiring oxygen supplementation. The median age of the cohort was 53 years (IQR 44-64), 730 (62\%) were male, and $882(75 \%)$ had at least 1 comorbidity. Of the total patients, $443(38 \%)$ required admission to a CCU, $321(27 \%)$ of the total admitted population died, and $849(73 \%)$ were discharged home after improvement.

As shown in Figure 1, a total of 349 (30\%) patients had an AKI diagnosis. Of them, 224 (19\%) and 125 (11\%) were categorized as CA-AKI and HA-AKI, respectively. The cohort characteristics at the time of AKI diagnosis and at admission are summarized in Table 1 and online suppl. Tables 1 and 2 (for all online suppl. material, see www.karger.com/doi/10.1159/000513948). There were differences in multiple parameters among the CA-AKI, HA-AKI, and the no AKI groups (online suppl. Table 2). The timing of the initial development of AKI with respect to hospital admission is shown in online suppl. Figure 1.

\section{Community-Acquired AKI}

Among 224 (19\%) patients with CA-AKI at admission, AKI was categorized as stage- 1 , stage-2, and stage- 3 in 134 (60\%), 50 (22\%), and 40 (18\%) cases, respectively. After excluding patients with previous $\mathrm{CKD}$, AKI stage 1 , stage 2 , and stage 3 occurred in 124 (61\%), 46 (23\%), and $32(16 \%)$ cases, respectively. Compared to patients with HA-AKI, those with CA-AKI were older (61 years [IQR 49-70] vs. 50 years [IQR 43-61]), had a higher number of comorbidities (diabetes [ 41 vs. $30 \%$ ], hypertension [ 44 vs. $26 \%$ ], CKD [10 vs. 3\%]), higher Charlson Comorbidity Index (2 points [IQR 1-4] vs. 1 point [IQR 0-2]), and presented to the emergency department with more severe COVID-19 disease parameters, including the $\mathrm{PaO}_{2}$ to $\mathrm{FiO}_{2}(\mathrm{P} / \mathrm{F})$ ratio (159 mm Hg [IQR 100-236] vs. 170 mm Hg [IQR 108252]), high-sensitivity C-reactive protein (20.2 mg/dL [IQR 13.8-29.3] vs. $18.4 \mathrm{mg} / \mathrm{dL}$ [IQR 12.5-25.0]), D-dimer (111 $\mathrm{ng} / \mathrm{mL}$ [IQR 718-2,418] vs. $732 \mathrm{ng} / \mathrm{mL}$ [IQR 532-1,427]), neutrophil to lymphocyte ratio (15.3 [IQR 8.4-24.4] vs. 10.9 [IQR 7.4-15.0]), SOFA score (4 points [IQR 3-5] vs. 3 points [IQR 2-4]), and a greater COVID-19 probability of ICU admission estimated by the ABC-GOALS score [24] (53\% [IQR 35-81] vs. 40\% [IQR 35-64]) (Table 1). 
Table 1. Characteristics of the patients at the time of AKI diagnosis and disease outcomes

\begin{tabular}{|c|c|c|c|}
\hline & CA-AKI $(n=224)$ & HA-AKI $(n=125)$ & $p$ value \\
\hline \multicolumn{4}{|l|}{ Demographics } \\
\hline Age, years & $61(49-71)$ & $50(43-61)$ & $<0.001$ \\
\hline Male, $n(\%)$ & $158(71)$ & $84(67)$ & 0.546 \\
\hline \multicolumn{4}{|l|}{ Comorbidities } \\
\hline Charlson Comorbidity Index, points & $2(1-4)$ & $1(0-2)$ & $<0.001$ \\
\hline Diabetes, $n(\%)$ & $90(40)$ & $39(31)$ & 0.106 \\
\hline Hypertension, $n(\%)$ & $100(45)$ & $31(25)$ & $<0.001$ \\
\hline Obesity, $n(\%)$ & $93(42)$ & $70(56)$ & 0.010 \\
\hline $\mathrm{CKD}, n(\%)$ & $22(10)$ & $3(2)$ & 0.015 \\
\hline \multicolumn{4}{|l|}{ Emergency room evaluation } \\
\hline Days from the start of symptoms & $7(5-11)$ & $7(6-9)$ & 0.180 \\
\hline Mean arterial pressure, $\mathrm{mm} \mathrm{Hg}$ & $86(76-97)$ & $90(81-97)$ & 0.011 \\
\hline Oxygen saturation, $n(\%)$ & $78(59-85)$ & $80(64-86)$ & 0.124 \\
\hline \multicolumn{4}{|l|}{ Laboratory at AKI detection } \\
\hline Total leukocytes, $\times 10^{3} / \mathrm{mm}^{3}$ & $10.4(7.6-15.0)$ & $10.1(8.00-13.0)$ & 0.558 \\
\hline Neutrophil to lymphocyte ratio & $15.3(8.43-24.4)$ & $8.42(5.18-12.7)$ & $<0.001$ \\
\hline Hemoglobin, g/dL & $15.0(13.6-16.3)$ & $13.1(11.7-14.8)$ & $<0.001$ \\
\hline Glucose, mg/dL & $160(116-235)$ & $132(101-176)$ & $<0.001$ \\
\hline Creatine kinase, U/L & $118(63-276)$ & $250(76-802)$ & $<0.001$ \\
\hline Lactate dehydrogenase, U/L & $467(337-629)$ & $428(318-548)$ & 0.116 \\
\hline C-reactive protein, $\mathrm{mg} / \mathrm{dL}$ & $20.2(13.8-29.5)$ & $19.5(7.8-30.1)$ & 0.141 \\
\hline Ferritin, ng/mL & $766(406-1,326)$ & $890(508-1,387)$ & 0.220 \\
\hline D-dimer, ng/mL & $1,120(718-2,502)$ & $2,398(1,087-5,451)$ & $<0.001$ \\
\hline Troponin I >ULN, $n(\%)$ & $82(37)$ & na & - \\
\hline $\mathrm{PaO}_{2} / \mathrm{FiO}_{2}$ ratio & $159(100-236)$ & $131(91-166)$ & $<0.001$ \\
\hline SOFA score, points & $4(3-5)$ & $7(6-9)$ & $<0.001$ \\
\hline \multicolumn{4}{|l|}{ Kidney function and critical support } \\
\hline Admission sCr, mg/dL & $1.5(1.3-2.0)$ & $1.0(0.8-1.2)$ & $<0.001$ \\
\hline sCr at $\mathrm{AKI}$ diagnosis, $\mathrm{mg} / \mathrm{dL}$ & $1.5(1.3-2.0)$ & $1.4(1.1-2.1)$ & 0.297 \\
\hline Peak sCr, mg/dL & $1.6(1.3-2.5)$ & $1.8(1.3-4.1)$ & 0.051 \\
\hline Discharge sCr, mg/dL & $1.0(0.8-1.6)$ & $1.1(0.7-2.3)$ & 0.207 \\
\hline \multicolumn{4}{|l|}{ AKI } \\
\hline Stage $1, n(\%)$ & $134(60)$ & $47(38)$ & $<0.001$ \\
\hline Stage $2, n(\%)$ & $50(22)$ & $30(24)$ & $<0.001$ \\
\hline Stage $3, n(\%)$ & $40(18)$ & $48(38)$ & $<0.001$ \\
\hline Days from admission to diagnosis & $0(0-0)$ & $4(2-8)$ & $<0.001$ \\
\hline Days from the start of symptoms & $7(5-11)$ & $13(9-19)$ & $<0.001$ \\
\hline $\mathrm{RRT}, n(\%)$ & $16(7)$ & $34(27)$ & $<0.001$ \\
\hline Intubated before AKI, $n(\%)$ & - & $109(87)$ & - \\
\hline Intubated at any time, $n(\%)$ & $66(30)$ & $110(88)$ & $<0.001$ \\
\hline Vasopressor before AKI, $n(\%)$ & - & $110(88)$ & - \\
\hline AKI resolved, $n(\%)$ & $167(75)$ & $74(59)$ & $<0.001$ \\
\hline \multicolumn{4}{|l|}{ Patient disposition } \\
\hline General ward, $n(\%)$ & $82(37)$ & $8(6)$ & $<0.001$ \\
\hline $\mathrm{CCU}, n(\%)$ & $142(63)$ & $117(94)$ & $<0.001$ \\
\hline \multicolumn{4}{|l|}{ Outcomes } \\
\hline Discharged, $n(\%)$ & $105(47)$ & $62(50)$ & 0.343 \\
\hline Length of hospitalization until discharge, days & $10(7-19)$ & $26(19-33)$ & $<0.001$ \\
\hline Death, $n(\%)$ & $119(53)$ & $63(50)$ & 0.656 \\
\hline Length of hospitalization until death, days & $4(2-8)$ & $9(5-17)$ & $<0.001$ \\
\hline
\end{tabular}

CA-AKI, community-acquired acute kidney injury; HA-AKI, hospital-acquired acute kidney injury; RAAS, renin-angiotensin-aldosterone system; RRT, renal replacement therapy; ARB, angiotensin receptor blockers; ULN, upper limit of normal; SOFA, sequential organ failure assessment score; CCU, critical care unit; na, not available; sCr, serum creatinine. 
Table 2. Urinalysis and urine electrolytes in the studied groups

\begin{tabular}{|c|c|c|c|c|}
\hline & No AKI $(n=158)$ & CA-AKI $(n=118)$ & HA-AKI $(n=87)$ & $p$ value \\
\hline \multicolumn{5}{|l|}{ Urinalysis } \\
\hline Specific gravity & $1.020(1.015-1.027)$ & $1.018(1.015-1.024)$ & $1.018(1.014-1.025)$ & 0.380 \\
\hline \multicolumn{5}{|l|}{ Proteinuria (dipstick) } \\
\hline Negative & $44(28)$ & $24(21)$ & $32(36)$ & \multirow{4}{*}{0.232} \\
\hline $1+$ & $58(37)$ & $45(39)$ & $26(29)$ & \\
\hline $2+$ & $47(30)$ & $40(35)$ & $29(32)$ & \\
\hline $3+$ & $9(6)$ & $6(5)$ & $3(3)$ & \\
\hline \multicolumn{5}{|l|}{ Hematuria (dipstick) } \\
\hline Negative & $102(65)$ & $53(46)$ & $36(40)$ & \multirow{4}{*}{$<0.001$} \\
\hline $1+$ & $35(22)$ & $28(24)$ & $19(21)$ & \\
\hline $2+$ & $12(8)$ & $23(20)$ & $20(22)$ & \\
\hline $3+$ & $9(6)$ & $11(10)$ & $13(14)$ & \\
\hline Leukocyturia, $n(\%)$ & $48(30)$ & $52(45)$ & $57(63)$ & $<0.001$ \\
\hline Hematuria (sediment), $n(\%)$ & $33(21)$ & $32(28)$ & $52(58)$ & $<0.001$ \\
\hline \multicolumn{5}{|l|}{ Urine electrolytes } \\
\hline Urine sodium, mmol/L & $28(15-46)$ & $27(16-43)$ & $22(12-44)$ & \multirow[t]{2}{*}{0.532} \\
\hline $\mathrm{U}_{\mathrm{Na}}<20 \mathrm{mmol} / \mathrm{L}, n(\%)$ & $24(37)$ & $34(43)$ & $36(32)$ & \\
\hline $\mathrm{U}_{\mathrm{Na}} 20-40 \mathrm{mmol} / \mathrm{L}, n(\%)$ & $20(31)$ & $21(27)$ & $82(32)$ & \multirow[t]{2}{*}{0.555} \\
\hline $\mathrm{U}_{\mathrm{Na}}>40 \mathrm{mmol} / \mathrm{L}, n(\%)$ & $21(32)$ & $24(30)$ & $61(32)$ & \\
\hline FE sodium, $\%$ & $0.17(0.08-0.45)$ & $0.30(0.12-0.62)$ & $0.22(0.14-0.62)$ & 0.046 \\
\hline $\mathrm{FE}_{\mathrm{Na}}<0.5 \%, n(\%)$ & $45(78)$ & $53(71)$ & $75(71)$ & \multirow{4}{*}{0.021} \\
\hline $\mathrm{FE}_{\mathrm{Na}} 0.5-1.0 \%, n(\%)$ & $9(16)$ & $13(17)$ & $10(9)$ & \\
\hline $\mathrm{FE}_{\mathrm{Na}} 1.0-2.0 \%, n(\%)$ & $4(7)$ & $2(3)$ & $14(13)$ & \\
\hline $\mathrm{FE}_{\mathrm{Na}}>2 \%, n(\%)$ & $0(0)$ & $7(9)$ & $7(7)$ & \\
\hline
\end{tabular}

CA-AKI, community-acquired acute kidney injury; HA-AKI, hospital-acquired acute kidney injury; FE, fractional excretion.

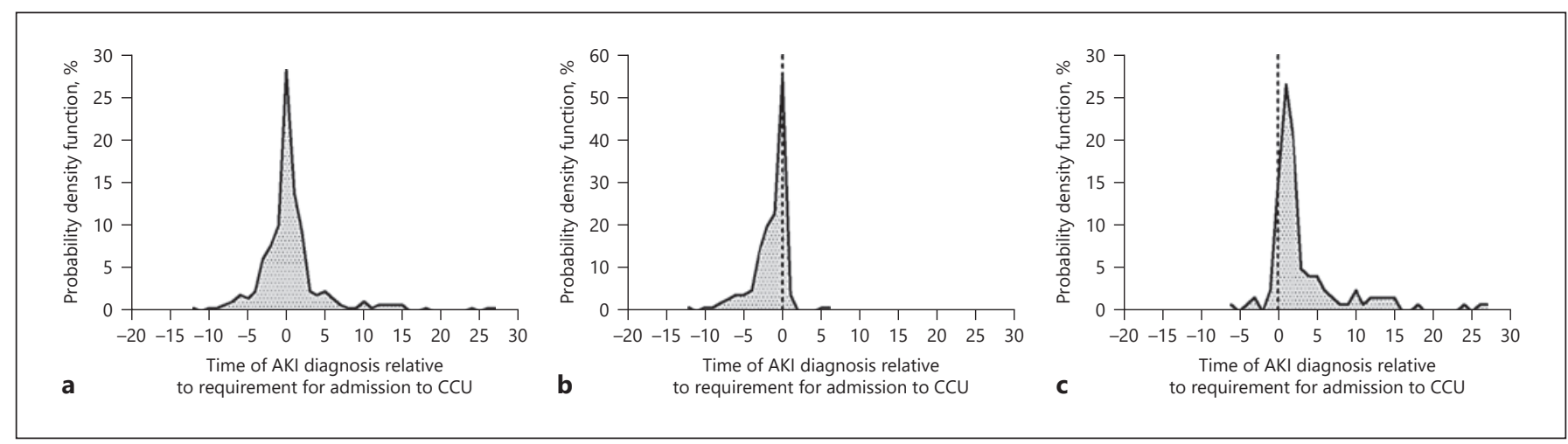

Fig. 2. Timing of CCU admission regarding AKI in all patients (a), CA-AKI (b), and HA-AKI (c). HA-AKI, hospital-acquired acute kidney injury; CA-AKI, community-acquired acute kidney injury; CCU, critical care unit.

A total of 118 (53\%) of CA-AKI cases had a urinalysis and urine electrolytes evaluation within $24 \mathrm{~h}$ of the AKI episode. As shown in Table 2, sodium fractional excretion (FE) was $<0.5 \%$ in 75 (71\%) cases, and urinary abnormalities such as hematuria and proteinuria $\geq 2+$ were observed in 33 (28\%) and 46 (39\%) patients, respectively.
After excluding patients with evidence of CKD, microscopic hematuria ( $n=30 / 102,29 \%)$ and proteinuria $\geq 2$ ( $n=37 / 102,36 \%)$ were frequent findings in CA-AKI.

Among patients with CA-AKI, a higher number of patients required admission to CCU than in the groups of patients without AKI (142 [63\%] vs. 184 [22\%], $p<$ 
Fig. 3. Outcomes by stages of AKI. HAAKI, hospital-acquired acute kidney injury; CA-AKI, community-acquired acute kidney injury.

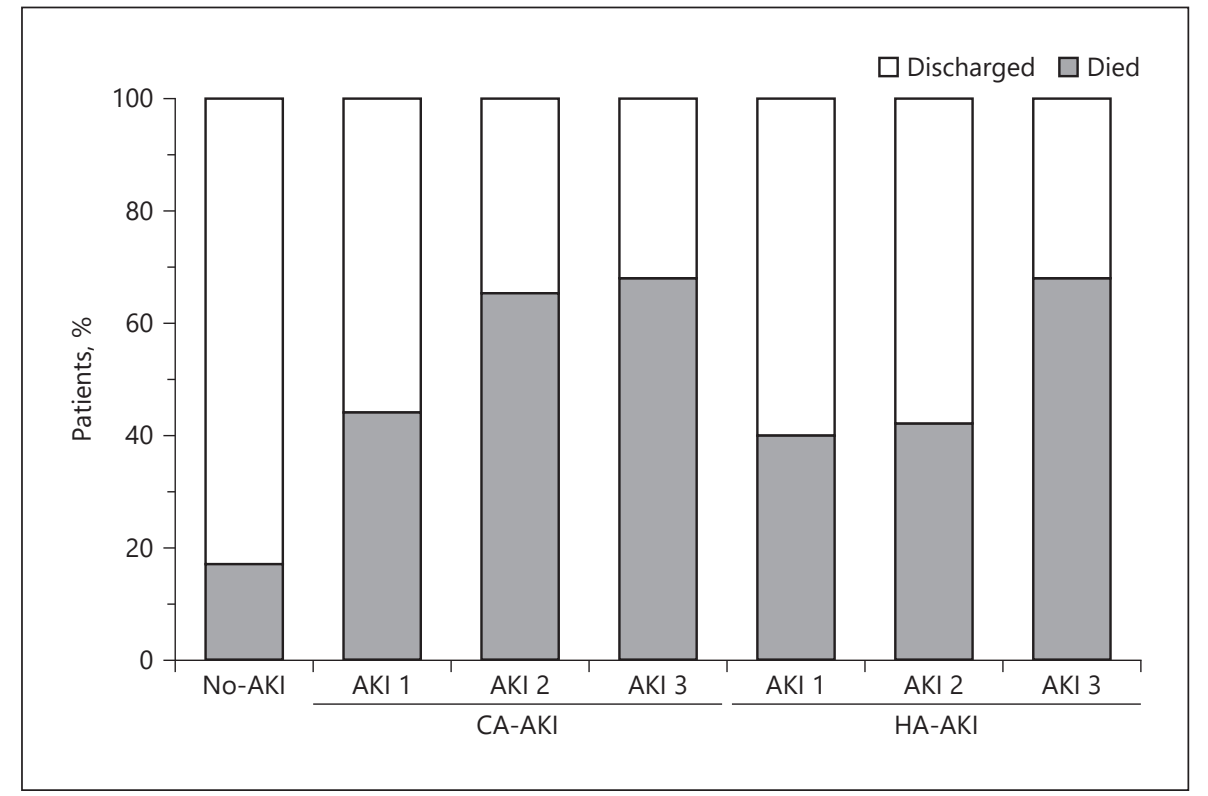

Table 3. Factors associated with CA-AKI and HA-AKI

\begin{tabular}{|c|c|c|c|c|}
\hline & \multicolumn{2}{|l|}{ Bivariate } & \multicolumn{2}{|l|}{ Multivariate } \\
\hline & OR $(95 \% \mathrm{CI})$ & $p$ value & $\operatorname{HR}(95 \% \mathrm{CI})$ & $p$ value \\
\hline \multicolumn{5}{|l|}{ CA-AKI } \\
\hline Charlson Comorbidity Index, per point & $1.47(1.34-1.61)$ & $<0.001$ & $1.16(1.02-1.32)$ & 0.020 \\
\hline CKD, versus no & $8.02(3.83-16.8)$ & $<0.001$ & $4.17(1.53-11.3)$ & 0.005 \\
\hline Hypertension, versus no & $2.44(1.79-3.32)$ & $<0.001$ & $1.55(1.01-2.36)$ & 0.043 \\
\hline SOFA score, per point & $2.71(2.34-3.14)$ & $<0.001$ & $2.19(1.87-2.57)$ & $<0.001$ \\
\hline Glucose at admission, per $10 \mathrm{mg} / \mathrm{dL}$ & $1.07(1.05-1.08)$ & $<0.001$ & $1.04(1.03-1.06)$ & $<0.001$ \\
\hline Creatine kinase, per $10 \mathrm{U} / \mathrm{dL}$ & $1.05(1.02-1.09)$ & 0.005 & $1.06(1.01-1.12)$ & 0.021 \\
\hline C-reactive protein, per $\mathrm{mg} / \mathrm{dL}$ & $1.08(1.06-1.09)$ & $<0.001$ & $1.04(1.02-1.06)$ & $<0.001$ \\
\hline Troponin I > ULN, versus no & $5.41(3.78-7.75)$ & $<0.001$ & $1.91(1.20-3.05)$ & 0.006 \\
\hline \multicolumn{5}{|l|}{ HA-AKI } \\
\hline BMI, per $\mathrm{kg} / \mathrm{m}^{2}$ & $1.07(1.04-1.10)$ & $<0.001$ & $1.04(0.99-1.09)$ & 0.053 \\
\hline Glucose at admission, per $10 \mathrm{mg} / \mathrm{dL}$ & $1.05(1.03-1.07)$ & $<0.001$ & $1.05(1.02-1.08)$ & 0.002 \\
\hline Troponin I >ULN, versus no & $3.61(2.29-5.69)$ & $<0.001$ & $1.95(1.01-3.83)$ & 0.046 \\
\hline Intubation, versus no & $77.5(42.9-140)$ & $<0.001$ & $68.2(37.1-126)$ & $<0.001$ \\
\hline
\end{tabular}

$\mathrm{ARB}$, angiotensin receptor blocker; CA-AKI, community-acquired acute kidney injury; HA-AKI, hospitalacquired acute kidney injury; SOFA, sequential organ failure assessment score; ULN, upper limit of normal.

0.001). Of these CA-AKI patients, $115(81 \%)$ required admission within $72 \mathrm{~h}$ following AKI detection (Fig. 2). Renal replacement therapy (RRT) was required in 16 cases (7.1\%). Of these, 3 (19\%) died and 13 (81\%) evolved favorably and were discharged. At the time of discharge, 5/13 (38\%) still required RRT, and 8/13 (62\%) recovered kidney function. The main indications for RRT were volume management (63\%), hyperkalemia (25\%), and acidosis (12\%). In 167 (75\%) patients, the AKI event had resolved by their last follow-up; nevertheless, mortality was higher for each progressive AKI stage and much higher than in patients without AKI (Fig. 3). Among patients discharged from hospitalization, those who experienced stage 3 AKI had a numerically higher $(p=0.096)$ sCr $(1.3 \mathrm{mg} / \mathrm{dL}, \mathrm{IQR} 0.7-2.7)$ than patients with stage 1 $(0.9 \mathrm{mg} / \mathrm{dL}, \mathrm{IQR} 0.7-1.1)$ or stage $2(0.8 \mathrm{mg} / \mathrm{dL}, \mathrm{IQR} 0.6-$ $0.9) \mathrm{AKI}$, and patients without AKI $(0.8 \mathrm{mg} / \mathrm{dL}, \mathrm{IQR} 0.7-$ 0.9) (Fig. 4). 


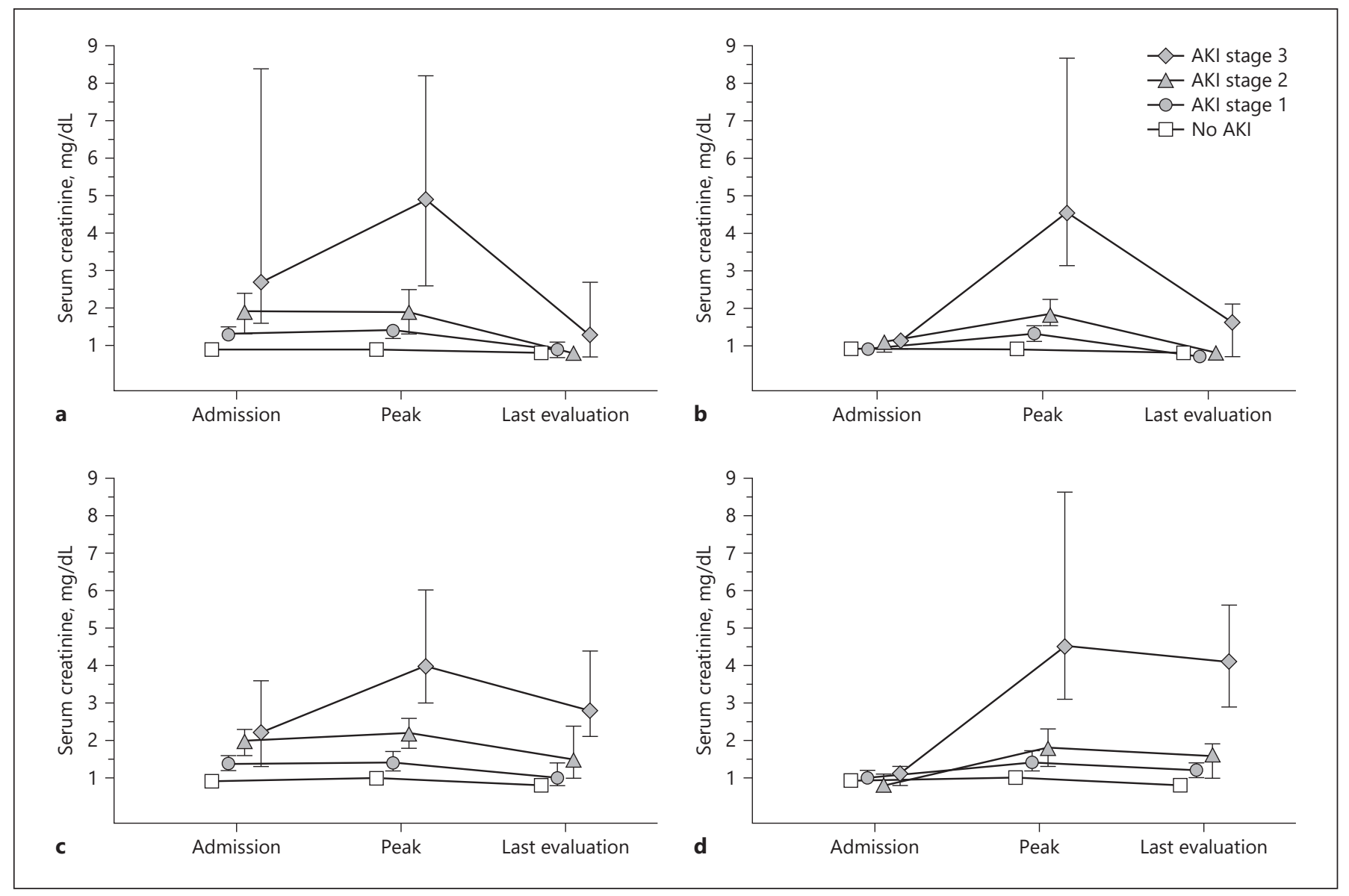

Fig. 4. Admission, peak, and last evaluation $\mathrm{sCr}$ in survivors (a, b) and non-survivors (c, d) with CA-AKI (a, c) and HA-AKI (b, d). * The marker represents the median and the lines the interquartile range. HA-AKI, hospitalacquired acute kidney injury; CA-AKI, community-acquired acute kidney injury; sCr, serum creatinine.

Factors Associated with CA-AKI

Several factors were associated with CA-AKI in the bivariate logistic regression analysis (Table 3 ). In the multivariate analysis, the Charlson Comorbidity Index (OR $1.16,95 \%$ CI 1.02-1.32), a previous history of CKD (OR 4.17 , 95\% CI 1.53-11.3), and prior hypertension diagnosis (OR 1.55, 95\% CI 1.01-2.36) were associated with CAAKI. Furthermore, several biomarkers of COVID-19 disease severity at admission were associated with CA-AKI, including the SOFA score (OR 2.19, 95\% CI 1.87-2.57), glucose levels (OR 1.04, 95\% CI 1.03-1.06), creatine phosphokinase (OR 1.06, 95\% CI 1.01-1.12), C-reactive protein (OR 1.04, 95\% CI 1.02-1.06), and troponin I serum levels above the upper reference level (OR 1.91, 95\% CI 1.20-3.05) (Table 3).

\section{Hospital-Acquired AKI}

HA-AKI was observed in $125(11 \%)$ patients. AKI staging was categorized as 1,2 , and 3, in $47(38 \%), 30$ (24\%), and 48 (38\%) patients, respectively. After excluding patients with previous $\mathrm{CKD}, \mathrm{AKI}$ stage 1 , stage 2 , and stage 3 occurred in 46 (38\%), 30 (25\%), and $46(38 \%)$ cases, respectively. In contrast to what was observed in patients with CA-AKI, those with HA-AKI had similar age, Charlson Comorbidity Index, the prevalence of CKD, or previous use of angiotensin receptor blockers when compared to patients who did not develop AKI during their disease course. Compared to the group without AKI, HAAKI patients had higher rates of obesity and higher disease severity parameters at admission, including the SOFA score, neutrophil to lymphocyte ratio, serum glucose, lactate dehydrogenase, C-reactive protein, troponin, D-dimer, and $\mathrm{P} / \mathrm{F}$ ratio (online suppl. Table 1). 
At the time of AKI diagnosis, inflammatory parameters and disease severity scores were higher in patients with HA-AKI than in patients with CA-AKI. These included higher SOFA score, lymphocyte count, serum glucose, creatine kinase, D-dimer, and lower P/F and N/L ratios and hemoglobin (Table 1). Compared to patients with CA-AKI, urinalysis performed for AKI evaluation in HA-AKI patients had a higher prevalence of hematuria (59 vs. $28 \%$ ), but similar proportions of proteinuria $>2+$ (34 vs. $41 \%$ ) and $\mathrm{FE}$ of sodium $<0.5 \%$ (71 vs. $71 \%$ ) (Table 2).

By the time of HA-AKI diagnosis, 109/125 (87\%) patients were already on mechanical ventilation and $110 / 125$ $(88 \%)$ were receiving vasopressor therapy. HA-AKI developed within $72 \mathrm{~h}$ of intubation in 78 (67\%) patients (Fig. 2).

RRT was required by 34 (27\%) HA-AKI patients. The main indications for RRT were volume management $(88 \%)$ and hyperkalemia (12\%). Eighteen (53\%) patients died and $16(47 \%)$ were discharged. At the time of discharge, 15/16 (94\%) recovered enough kidney function to suspend RRT and 1/16 (6\%) was still under RRT. Within the whole HA-AKI group, 74 (59\%) resolved their AKI by their last observation. Among patients discharged from hospitalization, the $\mathrm{sCr}$ at discharge was significantly higher $(p=0.008)$ in patients who experienced stage 3 AKI $(1.6 \mathrm{mg} / \mathrm{dL}$, IQR $0.7-2.1)$ than in patients with stage $1(0.7 \mathrm{mg} / \mathrm{dL}$, IQR $0.6-0.8)$ or stage $2(0.8 \mathrm{mg} / \mathrm{dL}$, IQR 0.6-0.9) AKI, and patients without AKI (0.8 mg/dL, IQR $0.7-0.9$ ) (Fig. 4). Mortality increased by each progressive stage of HA-AKI but was not different than in the CAAKI group (Fig. 3).

\section{Factors Associated with HA-AKI Development}

When HA-AKI was compared to CA-AKI, the Charlson Comorbidity Index, age, history of $\mathrm{CKD}$, and prior diagnosis of hypertension were not found to be significant in the bivariate logistic regression analysis. In the multivariate analysis, intubation (OR 68.2, 95\% CI 37.1126), cardiac troponin I levels above the upper reference limit (OR 1.95, 95\% CI 1.01-3.83), and glucose at admission (OR 1.05, 95\% CI 1.02-1.08) were independently associated with HA-AKI development (Table 3).

\section{Discussion/Conclusion}

As the COVID-19 pandemic evolves, it is becoming more evident that AKI is a common event in hospitalized COVID-19 patients. In this study, we observed that both
CA-AKI and HA-AKI are strong indicators of severe COVID-19 and are associated with mortality rates above $50 \%$. Nevertheless, there were clear distinctions between CA-AKI and HA-AKI [1]: CA-AKI proportion was almost double yet less severe than HA-AKI (19 vs. 11\%) [2]; CA-AKI occurred in patients with less "renal resilience" and high susceptibility to acute insults, as it was observed more frequently in older patients, with a high proportion of comorbidities as diabetes, CKD, and hypertension [3]; HA-AKI developed more commonly in younger patients without comorbidities, appeared suddenly as a stage 3 AKI in half of the cases after 2-8 days of hospitalization, and was accompanied by severe organ dysfunction assessed by the SOFA score, commonly with severe pneumonia requiring invasive mechanical ventilation [4]; both AKI classes occurred along with an intense inflammatory response and close to admission to the CCU.

AKI occurs in $8.4-46 \%$ of COVID-19 patients [1214 ], with incidences up to $75 \%$ in patients admitted to a CCU [14-16]. However, the exact incidence of AKI associated with COVID-19 is imprecise and inconstant, as depends on illness severity, study design, selected population, and the site of AKI occurrence (e.g., CCU, general hospital wards, and ambulatory clinic).

In many epidemiological studies, the global incidence of CA-AKI in non-COVID-19 illness appears to be rising, especially in older populations with chronic comorbidities $[25,26]$. In a recent report, the occurrence of CA-AKI versus HA-AKI in COVID-19 patients was estimated to occur in a proportion between 2:1 and 3:1 [13,27], similar to our results. We propose that the categorization of CAAKI and HA-AKI may be useful to understand the variable AKI rates reported in COVID-19.

It is extensively known that AKI development is associated with higher mortality risk, with rates of up to 40 $50 \%$ in non-COVID-19 patients with acute respiratory distress syndrome $[28,29]$. Specifically, AKI associated with COVID-19 has been linked to a longer duration of mechanical ventilation, length of hospitalization stay, and a greater mortality risk [16]. The convenience to categorize AKI into CA-AKI and HA-AKI has been put into question, given that both entities are supposedly associated with similar long-term sequelae and mortality [30, 31]. However, 2 previous meta-analyses suggested that CA-AKI has a better prognosis than HA-AKI, with a lower incidence of oliguria, reduced risk for CCU admission, and shorter hospital stay [18, 32]. In this cohort, we observed that patients with HA-AKI had a fourfold longer hospitalization stay, a greater need for RRT, and a lower proportion of renal function recovery. Moreover, both 
CA-AKI and HA-AKI were associated with similar mortality rates. A recent Spanish cohort of COVID-19 patients reported the same finding [33]. We hypothesize that older patients, with higher comorbidity burden, present sooner with kidney failure, but they are also more likely to die rapidly from severe COVID-19. Conversely, younger patients, with fewer comorbidities and higher resilience, may survive longer and develop severe AKI once severe multiorgan disease is present. It is also possible that treatment and invasive support may have been limited in a proportion of patients with CA-AKI due to their previous medical conditions or due to limited critical care area availability.

Several parameters reflecting COVID-19-induced inflammation have been associated with prognosis. These parameters include the serum levels of C-reactive protein, lactate dehydrogenase, ferritin, procalcitonin, a high neutrophil to lymphocyte ratio, along with biomarkers of organ dysfunction such as cardiac-specific troponin I or biomarkers of oxygenation $[34,35]$. All these parameters along with higher SOFA scores were frequently observed in both forms of AKI. For patients with CA-AKI, these parameters were elevated from admission, while in patients with HA-AKI, these increased from admission to the time of AKI development.

As reported here and in other studies [13, 14], the main factor associated with HA-AKI in COVID-19 is the need for intubation and mechanical ventilation. Acute respiratory distress syndrome may induce AKI through several mechanisms, among which one important is the production of inflammatory cytokines, such as interleukin-6 [36, 37]. In a systematic review and meta-analysis, van den Akker et al. [38] found that the pooled odds ratio for AKI in mechanically ventilated patients was 3.16. Moreover, AKI development also worsens lung injury by perpetuating an inflammatory environment, promoting immune cell dysfunction and fluid overload, among other mechanisms [39].

The precise etiology of AKI in our cohort could not be fully elucidated and given the diversity of presentation is probably diverse and, in many cases multifactorial. Sepsis and hemodynamic instability may have a predominant role in HA-AKI cases, given that $80 \%$ of patients in the HA-AKI were under vasopressors by the time of AKI diagnosis. Microcirculatory dysfunction in sepsis due to the local and systemic release of pro-inflammatory cytokines, such as IL- 6 and TNF- $\alpha$, may induce intrarenal hemodynamic changes, including afferent arteriole vasoconstriction [40-42]. In a previous study, $65 \%$ of AKI episodes occurred in COVID-19 patients under vasopressor treat- ment, and the etiology was ascribed to be acute tubular injury [13]. In biopsy studies, almost all patients have been reported to have variable degrees of acute tubular injury, and $40-61 \%$ of them received vasopressor therapy. Interestingly, we found a FE of sodium below $1 \%$ at AKI diagnosis in 88 and $80 \%$ of patients with CA-AKI and HA-AKI, respectively, suggesting glomerular hypoperfusion and at least partially conserved tubular sodium absorption, without a full classic acute tubular injury. These results contrast a FE of sodium $<1 \%$ prevalence of $38 \%$ reported in a previous study in COVID-19 patients [13].

In addition to the tubular damage commonly described in critically ill patients, other mechanisms may contribute to kidney damage, such as inflammatory nephritis [43, 44], vascular microthrombosis [45], or subclinical glomerulopathy, among others. As reported here and previously, dipstick hematuria and proteinuria are frequent findings in COVID-19 patients. A proteinuria $>2+$ was observed in 41 and $34 \%$ of patients with CA-AKI and HA-AKI, respectively. These proteinuria percentages remained similar after excluding CKD cases and were similar to the prevalence of $39-44 \%$ in previous series [13, 46]. Proteinuria is hypothesized to be secondary to capillary leakage and/or decreased proximal tubular recapture [47], although we could not rule out a glomerular origin. Hematuria is also frequently reported, with a prevalence of 28 and 59\% observed in CA-AKI and HA-AKI. Cheng et al. [46] observed a comparable percentage of hematuria, of $37 \%$. It will probably be necessary to perform more kidney biopsies, even in the postmortem period, to assess if hematuria is a marker of more severe COVID-19 or if this indicates a subgroup of patients with direct damage to kidney tissue by SARS-CoV-2.

Our study has limitations. Even when the data were prospectively collected, we were not able to register the nephrology consultant's specific diagnoses. Urine sediment analysis was based on the automated analysis and not on visual evaluation by the nephrologist. AKI was exclusively defined and staged by sCr criteria as information on urine output was not available, thus the true AKI prevalence is likely to be higher in our population. Treatment data were not included in the analyses as most patients were included in randomized blinded clinical trials.

In conclusion, both CA-AKI and HA-AKI portend an adverse prognosis in COVID-19 patients. CA-AKI was associated with a higher comorbidity burden (including CKD and hypertension) and COVID-19 disease severity, while HA-AKI occurred in younger patients along with severe multiorganic disease and respiratory failure. 


\section{Statement of Ethics}

This study was approved by our local Research and Ethics Boards (reference number NMM-3325-20-20-1) and was conducted ethically in accordance with the World Medical Association Declaration of Helsinki.

\section{Conflict of Interest Statement}

The authors have nothing to disclose.

\section{Funding Sources}

This study was performed with local funds of the Department of Nephrology and Mineral Metabolism, Instituto Nacional de Ciencias Médicas y Nutrición Salvador Zubirán, Mexico City, Mexico.

\section{Author Contributions}

Conception and study design: A.J.M.R., J.M.M.V., M.A.H., R.C.R., and O.V.V. Data acquisition: A.J.M.R., R.D.A., R.A.M.P., D.A.F.C., J.E.G.A., N.B.P., A.F.C., R.A.C.B., and J.M.M.V. Data cleaning and preparation of the final database: A.M.J.R., R.D.A., R.A.M.P., D.A.F.C., and J.M.M.V. Analysis and interpretation of data: J.M.M.V., M.A.H., J.C.R.S., R.C.R., and O.V.V. Drafting and revising of the manuscript: all authors.

\section{References}

1 Helmy YA, Fawzy M, Elaswad A, Sobieh A, Kenney SP, Shehata AA. The COVID-19 pandemic: a comprehensive review of taxonomy, genetics, epidemiology, diagnosis, treatment, and control. J Clin Med. 2020;9(4):1225

2 Li W, Moore MJ, Vasilieva N, Sui J, Wong SK, Berne MA, et al. Angiotensin-converting enzyme 2 is a functional receptor for the SARS coronavirus. Nature. 2003;426(6965):450-4.

3 Diao B, Wang C, Wang R, Feng Z, Tan Y, Wang $\mathrm{H}$, et al. Human kidney is a target for novel severe acute respiratory syndrome coronavirus 2 (SARS-CoV-2) infection. 2020. www.medrxiv.org.https://doi.org/10.1101/20 20.03.04.20031120.

4 Su H, Yang M, Wan C, Yi LX, Tang F, Zhu $\mathrm{HY}$, et al. Renal histopathological analysis of 26 postmortem findings of patients with $\mathrm{CO}$ VID-19 in China. Kidney Int. 2020;98(1): 219-27.

5 Kudose S, Batal I, Santoriello D, Xu K, Barasch J, Peleg Y, et al. Kidney biopsy findings in patients with COVID-19. J Am Soc Nephrol. 2020;31(9):1959-68.

6 Santoriello D, Khairallah P, Bomback AS, Xu $\mathrm{K}$, Kudose S, Batal I, et al. Postmortem kidney pathology findings in patients with $\mathrm{CO}$ VID-19. J Am Soc Nephrol. 2020;31(9):215867.

7 Rossi GM, Delsante M, Pilato FP, Gnetti L, Gabrielli L, Rossini G, et al. Kidney biopsy findings in a critically ill COVID-19 patient with dialysis-dependent acute kidney injury: a case against "SARS-CoV-2 nephropathy". Kidney Int Rep. 2020;5(7):1100-5.

8 Sharma P, Uppal NN, Wanchoo R, Shah HH, Yang Y, Parikh R, et al. COVID-19-associated kidney injury: a case series of kidney biopsy findings. J Am Soc Nephrol. 2020;31(9): 1948-58.

9 Naicker S, Yang CW, Hwang SJ, Liu BC, Chen JH, Jha V. The novel coronavirus 2019 epidemic and kidneys. Kidney Int. 2020;97(5): $824-8$.
10 Perico L, Benigni A, Remuzzi G. Should COVID-19 concern nephrologists? why and to what extent? the emerging impasse of angiotensin blockade. Nephron. 2020;144(5):21321.

11 Martinez-Rojas MA, Vega-Vega O, Bobadilla NA. Is the kidney a target of SARS-CoV-2? Am J Physiol Renal Physiol. 2020;318(6): F1454-62.

12 Robbins-Juarez SY, Qian L, King KL, Stevens JS, Husain SA, Radhakrishnan J, et al. Outcomes for patients with COVID-19 and acute kidney injury: a systematic review and metaanalysis. Kidney Int Rep. 2020;5(8):1149-60.

13 Mohamed MMB, Lukitsch I, Torres-Ortiz AE, Walker JB, Varghese V, Hernandez-Arroyo $\mathrm{CF}$, et al. Acute kidney injury associated with coronavirus disease 2019 in Urban new Orleans. Kidney360. 2020;1(7):614-22.

14 Hirsch JS, Ng JH, Ross DW, Sharma P, Shah $\mathrm{HH}$, Barnett RL, et al. Acute kidney injury in patients hospitalized with COVID-19. Kidney Int. 2020;98(1):209-18.

15 Fisher M, Neugarten J, Bellin E, Yunes M Stahl L, Johns TS, et al. AKI in hospitalized patients with and without COVID-19: a Comparison Study. J Am Soc Nephrol. 2020; 31(9):2145-57.

16 Fominskiy EV, Scandroglio AM, Monti G, Calabrò MG, Landoni G, Dell'Acqua A, et al. Prevalence, characteristics, risk factors, and outcomes of invasively ventilated COVID-19 patients with acute kidney injury and renal replacement therapy. Blood Purif. 2021;50(1): 102-9.

17 Kidney Disease. Improving Global Outcomes (KDIGO) Acute Kidney Injury Work Group. KDIGO clinical practice guideline for acute kidney injury. Kidney Int Suppl. 2012;2:1138.
18 Inokuchi R, Hara Y, Yasuda H, Itami N, Terada Y, Doi K. Differences in characteristics and outcomes between community- and hospitalacquired acute kidney injury: a systematic review and meta-analysis. Clin Nephrol. 2017; 88(10):167-82.

19 Siew ED, Matheny ME. Choice of reference serum creatinine in defining acute kidney injury. Nephron. 2015;131(2):107-12.

20 KDIGO. Kidney disease improving global outcomes 2012 clinical practice guideline for the evaluation and management of chronic kidney disease. Kidney Int Suppl. 2013;3:1.

21 Levey AS, Stevens LA. Estimating GFR Using the CKD epidemiology collaboration (CKDEPI) creatinine equation: more accurate GFR estimates, lower CKD prevalence estimates, and better risk predictions. Am J Kidney Dis. 2010;55(4):622-7.

22 Chawla LS, Bellomo R, Bihorac A, Goldstein SL, Siew ED, Bagshaw SM, et al. Acute kidney disease and renal recovery: consensus report of the Acute Disease Quality Initiative (ADQI) 16 Workgroup. Nat Rev Nephrol. 2017;13(4):241-57.

23 Charlson ME, Pompei P, Ales KL, MacKenzie CR. A new method of classifying prognostic comorbidity in longitudinal studies: development and validation. J Chronic Dis. 1987; 40(5):373-83.

24 Mejia-Vilet JM, Córdova-Sánchez BM, Fernández-Camargo DA, Méndez-Pérez RA, Morales-Buenrostro LE, Hernández-Gilsoul T. A risk score to predict admission to the intensive care unit in patients with Covid-19: the ABC-GOALS score. Salud Publica Mex. 2021;63(1):1-11.

25 Hsu CN, Lee CT, Su CH, Wang YC, Chen HL, Chuang $\mathrm{JH}$, et al. Incidence, outcomes, and risk factors of community-acquired and hospital-acquired acute kidney injury: a retrospective Cohort Study. Medicine. 2016; 95(19):e3674. 
26 Yang L, Xing G, Wang L, Wu Y, Li S, Xu G, et al. Acute kidney injury in China: a cross-sectional survey. Lancet. 2015;386(10002):146571.

27 Pelayo J, Lo KB, Bhargav R, Gul F, Peterson E, DeJoy R III, et al. Clinical characteristics and outcomes of community- and hospital-acquired acute kidney injury with COVID-19 in a US Inner City hospital system. Cardiorenal Med. 2020;10(4):223-31.

28 Angus DC, Wax RS. Epidemiology of sepsis: an update. Crit Care Med. 2001;29(7 Suppl): S109-16.

29 Vincent JL, Sakr Y, Sprung CL, Ranieri VM, Reinhart K, Gerlach H, et al. Sepsis in European intensive care units: results of the SOAP study. Crit Care Med. 2006;34(2):344-53.

30 Daher EF, Silva Junior GB, Santos SQ, Bezerra CCR, Diniz EJ, Lima RS, et al. Differences in community, hospital and intensive care unit-acquired acute kidney injury: observational study in a nephrology service of a developing country. Clin Nephrol. 2012;78(6): 449-55.

31 Der Mesropian PJ, Kalamaras JS, Eisele G, Phelps KR, Asif A, Mathew RO. Long-term outcomes of community-acquired versus hospital-acquired acute kidney injury: a retrospective analysis. Clin Nephrol. 2014;81(3): $174-84$.

32 Huang L, Xue C, Kuai J, Ruan M, Yang B, Chen X, et al. Clinical characteristics and outcomes of community-acquired versus hospital-acquired acute kidney injury: a meta-analysis. Kidney Blood Press Res. 2019;44(5):87996.
33 Portolés J, Marques M, López-Sánchez P, de Valdenebro M, Muñez E, Serrano ML, et al. Chronic kidney disease and acute kidney injury in the COVID-19 Spanish outbreak. Nephrol Dial Transplant. 2020;35(8):135361.

34 Guan WJ, Ni ZY, Hu Y, Liang WH, Ou CQ, $\mathrm{He}$ JX, et al. Clinical characteristics of coronavirus disease 2019 in China. N Engl J Med. 2020;382(18):1708-20.

35 Chen R, Liang W, Jiang M, Guan W, Zhan C, Wang T, et al. Risk factors of fatal outcome in hospitalized subjects with coronavirus disease 2019 from a nationwide analysis in China. Chest. 2020;158(1):97-105.

36 Imai Y, Parodo J, Kajikawa O, de Perrot M, Fischer S, Edwards V, et al. Injurious mechanical ventilation and end-organ epithelial cell apoptosis and organ dysfunction in an experimental model of acute respiratory distress syndrome. JAMA. 2003;289(16):2104-12.

37 Liu KD, Glidden DV, Eisner MD, Parsons PE, Ware LB, Wheeler A, et al. Predictive and pathogenetic value of plasma biomarkers for acute kidney injury in patients with acute lung injury. Crit Care Med. 2007;35(12):2755-61.

38 van den Akker JP, Egal M, Groeneveld AB. Invasive mechanical ventilation as a risk factor for acute kidney injury in the critically ill: a systematic review and meta-analysis. Crit Care. 2013;17(3):R98.

39 Faubel S, Edelstein CL. Mechanisms and mediators of lung injury after acute kidney injury. Nat Rev Nephrol. 2016;12(1):48-60.

40 Benes J, Chvojka J, Sykora R, Radej J, Krouzecky A, Novak I, et al. Searching for mechanisms that matter in early septic acute kidney injury: an experimental study. Crit Care. 2011;15(5):R256.
41 Gomez H, Ince C, De Backer D, Pickkers P, Payen D, Hotchkiss J, et al. A unified theory of sepsis-induced acute kidney injury: inflammation, microcirculatory dysfunction, bioenergetics, and the tubular cell adaptation to injury. Shock. 2014;41(1):3-11.

42 Bhaskar S, Sinha A, Banach M, Mittoo S, Weissert R, Kass JS, et al. Cytokine storm in COVID-19: immunopathological mechanisms, clinical considerations, and therapeutic approaches: the REPROGRAM consortium position paper. Front Immunol. $2020 \mathrm{Jul}$ 10;11:1648.

43 Lerolle N, Nochy D, Guérot E, Bruneval P, Fagon JY, Diehl JL, et al. Histopathology of septic shock induced acute kidney injury: apoptosis and leukocytic infiltration. Intensive Care Med. 2010;36(3):471-8.

44 Aslan A, van den Heuvel MC, Stegeman CA, Popa ER, Leliveld AM, Molema G, et al. Kidney histopathology in lethal human sepsis. Crit Care. 2018;22(1):359.

45 Tavazzi G, Civardi L, Caneva L, Mongodi S, Mojoli F. Thrombotic events in SARS-CoV-2 patients: an urgent call for ultrasound screening. Intensive Care Med. 2020;46(6):1121-3.

46 Cheng Y, Luo R, Wang K, Zhang M, Wang Z, Dong $L$, et al. Kidney disease is associated with in-hospital death of patients with COVID-19. Kidney Int. 2020;97(5):829-38.

47 Werion A, Belkhir L, Perrot M, Schmit G, Aydin S, Chen Z, et al. SARS-CoV-2 causes a specific dysfunction of the kidney proximal Tubule. Kidney Int. 2020;98(5):1296-307. 